\title{
Lorentz Transmission Electron Microscopy Imaging of Magnetic Textures in MnBi
}

Núria Bagués ${ }^{1}$, Brandi L. Wooten ${ }^{2}$, Bin $\mathrm{He}^{3}$, Brian C. Sales ${ }^{4}$, Joseph Heremans ${ }^{5}$ and David McComb ${ }^{1}$

${ }^{1}$ Center for Electron Microscopy and Analysis, The Ohio State University, Columbus, OH, USA, COLUMBUS, Ohio, United States, ${ }^{2}$ Department of Materials Science and Engineering, The Ohio State University, Columbus, OH, USA, Columbus, Ohio, United States, ${ }^{3}$ Max Planck Insitute for Chemical Phisics of Solids, Dresden, Gemany, United States, ${ }^{4}$ Materials Science and Technology Division, Oak Ridge National Lab, Oak Ridge, TN, USA, United States, ${ }^{5}$ Department of Mechanical and Aerospace Engineering, The Ohio State University, Columbus, OH, USA, United States

$\mathrm{MnBi}$ has attracted much attention as a potential replacement for rare-earth magnets in engineering applications [1], as well as a system for exploration of exotic transport phenomena [2,3]. This ferromagnetic metal has a Curie temperature of $\sim 630 \mathrm{~K}$ and is a hard magnet at room temperature with the magnetic moments oriented along the c-axis of its NiAs-type hexagonal structure [2]. The magnetic moments rotate away from the c-axis below the initial spin reorientation temperature TSR $1=140 \mathrm{~K}$ and are oriented completely within the ab-plane below TSR2=90K [2]. Recent reports suggest that intriguing magnetic textures such as striped domains and skyrmion bubbles form in thin MnBi lamellae under certain temperature and magnetic field conditions [3].

In this contribution we present the results of an in-situ Lorentz transmission electron microscopy (TEM) study of magnetic textures in $\mathrm{MnBi}$. This contributes to a larger study exploring the influence of such textures on the thermal transport properties of $\mathrm{MnBi}$. For the TEM study, thin lamellae were extracted from $\mathrm{MnBi}$ single crystals along the ab-plane (with c-axis perpendicular to the long direction of the foil) using a focused ion beam (FIB) instrument. Since MnBi is sensitive to oxidation [2], the foils were transferred rapidly from the FIB to the TEM to minimise air exposure.

Initially, we performed annular dark field (ADF) imaging, energy dispersive x-ray (EDS) spectral mapping, and electron energy-loss (EELS) spectrum images of the thin lamellae. ADF images presented in Figure 1 show the hexagonal lattice observed with the c-axis out of the image (left image) as well as Moiré fringes consistent with the presence of oxidation products on the surface of the thin foil (right image).

The microscope was switched into Lorentz TEM mode, decreasing the in situ magnetic field applied to the foil from $\sim 2 \mathrm{~T}$ to 0T (with $\sim 10 \mathrm{mT}$ remnant field). The Lorentz TEM was performed using Fresnel imaging by collecting through-focal image series to observe the magnetic contrast. Using a double tilt cryogenic TEM specimen-holder enabled us to vary the sample temperature from $300 \mathrm{~K}$ to $90 \mathrm{~K}$. This temperature range was sufficient to track the same region of interest in the Lorentz TEM images through the spin re-orientation transition temperatures.

Under zero field cooling conditions, we observed spontaneous formation of stripe domains around 170K, which remained unchanged down to $90 \mathrm{~K}$. Surprisingly, these stripe domains were not present in the thinner regions of the lamellae. In contrast, imaging of a field cooled $(\sim 2 \mathrm{~T})$ sample revealed at $90 \mathrm{~K}$, when the field was removed, coexistence of stripe domains and magnetic bubbles, with the latter most abundant in the thick regions of the foil. The dependence of the magnetic textures in MnBi lamellae on temperature, 
applied field, and specimen thickness are currently under further investigation to establish the correlation with thermal transport properties in this system.
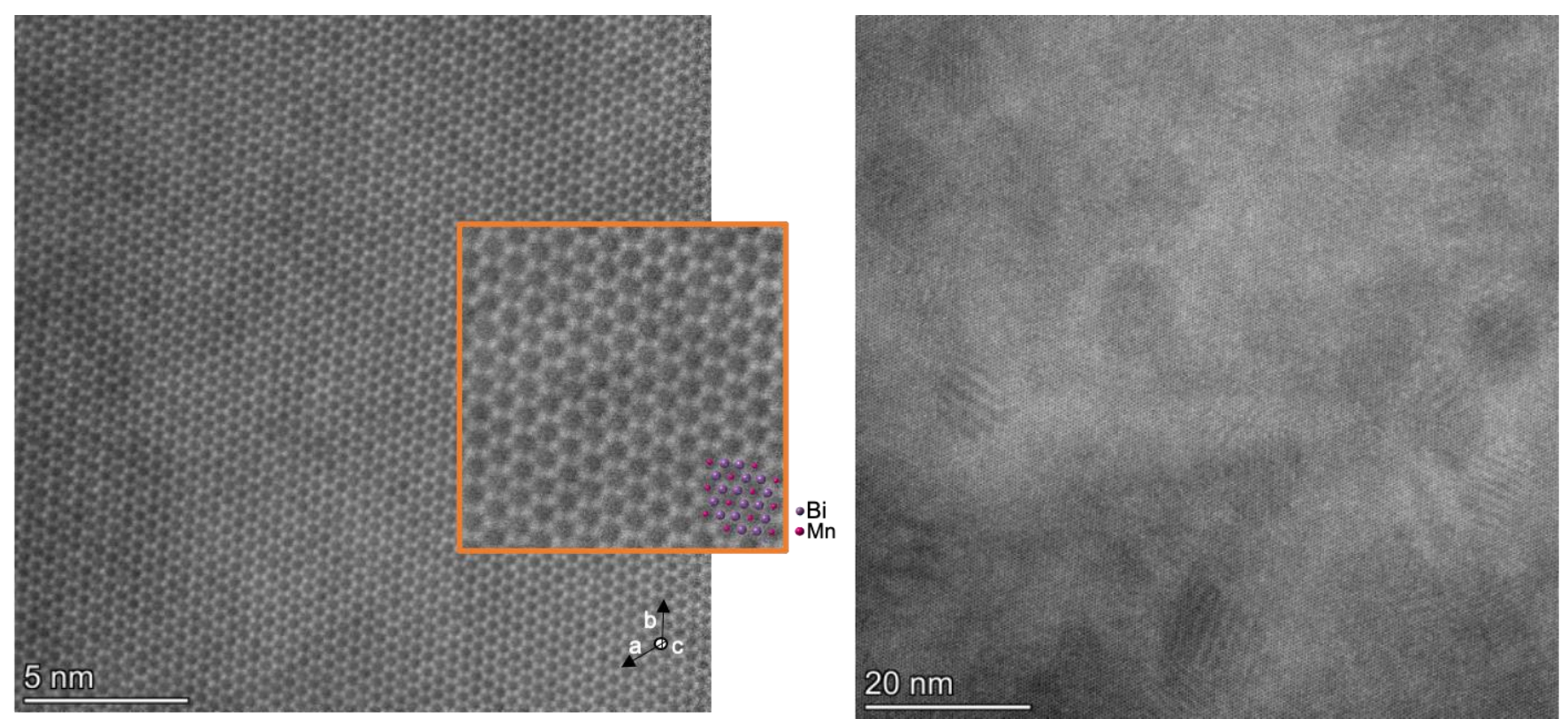

Figure 1. Figure 1. ADF images viewed along the c-axis of the MnBi. Left, high resolution image showing the hexagonal lattice. Right, low magnifications image showing the dark areas and Moiré fringes due to the presence of oxides on the surface of lamellae.

\section{References}

[1] N. V. Rama Rao et al., J. Phys. D: Appl. Phys. 46 (2013), 062001

[2] B. He et al., arXiv:2009.02211 (2020)

[3] Y. He et al., arXiv:2011.06340 (2020)

[4] M. A. McGuire et al., Physical Review B 90, (2014), 174425

[5] The authors acknowledge funding from Defense Advanced Research Projects Agency (DARPA) under Grant No. D18AP00008 and the Center for Emergent Materials at The Ohio State University, an NSF MRSEC (DMR-2011876). B.C.S. was supported by the DOE, Office of Science, Basic Energy Sciences, Materials Sciences and Engineering Division. The electron microscopy was performed at the Center for Electron Microscopy and Analysis (CEMAS) at The Ohio State University. 